\title{
Stability and change in the colour lexicon of the Japonic languages
}

\author{
John L.A. Huisman, Roeland van Hout and Asifa Majid \\ Radboud University | Radboud University | University of York
}

Previous work on colour lexicons focussed on universal patterns in their structure and evolution. We collected new colour naming data in Japanese and three under-described Ryukyuan languages (Amami, Miyako and Yayeama) to investigate semantic variation and change in the colour lexicon of related languages in a modern context. We found several new colours terms (e.g., midori and guriin for 'green') in the lexicon of Ryukyuan speakers, apparently resulting from contact with Standard Japanese and English. A comparison of our data with historical data suggests that modern Ryukyuan colour systems are closer to modern Japanese than they are to their historic pasts. However, we also found that modern-day Ryukyuan languages are more similar to each other than they are to Japanese. These findings show the scope of semantic changes that can occur through outside influence and highlight the need for fresh empirical data in the study of semantics in related languages.

Keywords: colour, semantics, language variation and change, Japonic, Ryukyuan

\section{General introduction}

As the world around us changes, our language changes with it. Technological and societal developments can increase the cultural salience of a concept and with that boost the need to talk about it. For example, as societies gain greater technological control of colour, the need to communicate about colour becomes more important and more terms are added to partition the colour spectrum (Berlin \& Kay 1969; Levinson 2000). For the colour lexicon, an immense body of workparticularly influenced by the classic study of Berlin \& Kay (1969) - has shown that its structure and evolution are cross-linguistically constrained. Several largescale projects, such as the World Color Survey (Kay et al. 1991) and the Mesoamerican Color Survey (MacLaury 1986), were launched to further test Berlin \& Kay's 
hypotheses across a wide range of languages, leading to refinements and additions to the original framework (see e.g., Kay et al. 1997; Kay et al. 2009; MacLaury et al. 1992; MacLaury 2001; Jäger 2012), as well as eliciting notable challenges (Lucy 1997; Levinson 2000; Wierzbicka 2008).

This prior work primarily focused on addressing the universality of constraints on the colour lexicon, so data collection was aimed towards lesserdescribed languages from a wide range of language families sampled from across the world to allow broad cross-linguistic comparison. Since typological studies in general tend to favour diverse language samples, the scope of semantic variation across related languages is often not well explored, and variation in colour terms and categories across related languages has received considerably less attention (although see, e.g., Kristol 1980; Majid et al. 2015; Haynie \& Bowern 2016; Lillo et al. 2018). In addition, it is notable that even modern explorations of colour language (e.g., Regier et al. 2007, 2009; Jäger 2012) are based on data that was collected several decades ago - surveys for both the World Color Survey and the Mesoamerican Color Survey were conducted in the 1970s.

This paper departs from this approach by collecting new data from speakers of several related unwritten languages, which are interesting to examine today because these communities have undergone many societal changes in recent times. Globalisation and interconnectedness have led to increased exposure to the influence of Western culture and the English language. Contact with other languages and cultures can lead to the introduction of new colour categories as speakers are exposed to colour system that differ from their own. For example, several languages in the World Color Survey (Kay et al. 2009) and the Mesoamerican Color Survey (MacLaury 1986) have forms of verde and azul for green and blue in their colour lexicon as a result of influence from Spanish.

The current study focuses on Japanese and the Ryukyuan languages spoken across the Japanese mainland and the Ryukyu Islands. The colour lexicon has been a topic of study in these languages since the 1960s (Kusakabe 1964; Berlin \& Kay 1969), but more recent work has mainly concerned Japanese (e.g., Uchikawa \& Boynton 1987; Kuriki et al. 2017). These later studies revealed several developments in the Japanese colour lexicon, but it is unclear to what extent the Ryukyuan languages have also changed. Given the societal changes and language contact in recent decades, the Ryukyu Islands make an ideal testbed to investigate the impact of modern society on these languages. Furthermore, the region provides an opportunity to investigate variation in the colour lexicon of a single language family - an understudied arena of the colour literature. Section 1.1 shortly introduces the language family itself and discusses some sociocultural differences between the Japanese mainland and the Ryukyu Islands, after which Section 1.2 
summarises previous work on colour vocabulary in the Japonic languages, and Section 1.3 introduces the current study.

\subsection{The Japonic language family}

The Japonic language family consists of two major branches. The first is the Japanese branch, comprising the varieties spoken on the main islands, which are generally subdivided into Eastern, Western, Kyushu, and Hachijo Japanese (Shibatani 1990). Modern Standard Japanese is a predominantly Eastern Japanese variety with some influences from Western Japanese (Iwasaki 2013). The second branch of the Japonic language family is Ryukyuan, which comprises the varieties spoken across the smaller islands in the south. Ryukyuan is generally subdivided into Amami and Okinawa (Northern Ryukyuan) on one hand, and Miyako, Yaeyama and Yonaguni (Southern Ryukyuan) on the other (Pellard 2015; Huisman et al. 2019).

Since the turn of the 2oth century, when the Ryukyu Islands were incorporated into Japan as a country, more and more people have been exposed to and eventually become bilingual in Standard Japanese in addition to their local Ryukyuan variety. Various language policies over the course of the 2oth century then diminished the overall use of Ryukyuan, resulting in Japanese becoming the first language for many speakers, and leading to the eventual endangered status for Ryukyuan in the 21st century, with no monolingual speakers remaining (Heinrich 2005).

While both Japanese and Ryukyuan have been intensively exposed to Western culture and the English language in the immediate post-war period, the Ryukyu Islands (Okinawa in particular) have their own unique linguistic situation as a result of prolonged American occupation, which led to the incorporation of English loanwords not found in Standard Japanese (Tsuhako 1992). In addition, the increased use of Standard Japanese across the Ryukyus means that speakers of Ryukyuan are now also exposed to English loanwords that have become integrated into the standard language.

In addition to the linguistic situation, there are also some cultural factors to take into consideration. First, as more elaborate material culture (e.g., increased and more specialised use of dyeing technology) is often associated with a larger colour lexicon (Naroll 1970; Ember 1978), it is relevant to note that there is little difference between the general levels of traditional material culture in Japan and the Ryukyus. Both regions have extensive dyeing practices with elaborate colour designs since the Middle Ages, seen in the Japanese kimono and Okinawa's counterpart bingata, for example. Next, it is also important to consider that the Ryukyu Islands were politically and culturally independent from mainland Japan 
for several centuries, including when Japan closed itself off from the outside world during the sakoku policy. The former Ryukyu Kingdom traded freely with countries across the region, allowing greater exposure to other cultures and languages with different technologies, concepts, and vocabulary.

This combination of cultural interest in colour through material culture and the prolonged contact with both Japanese and English would predict that the Ryukyuan languages have large colour lexicons comparable to the larger languages documented in the colour literature (e.g., Berlin \& Kay 1969), but this is not the case as will become clear in Section 1.2.

\subsection{Studies on colour in the Japonic language family}

There have been several studies on the colour lexicon of Standard Japanese. Its inclusion in the original study by Berlin and Kay (1969), combined with a now classic naming study by Uchikawa and Boynton (1987), followed by a further replication by Kuriki and colleagues (2017), provide us with around five decades of data on the Japanese colour lexicon. These studies reveal that the Japanese colour lexicon has seen changes within this period - see Table 1 for a summary.

Table 1. Japanese colour terms across three major colour chip naming studies

\begin{tabular}{llll}
\hline & B\&K & U\&B & Ket al. \\
\hline WHITE & shiro & shiro & shiro \\
GREY & haiiro / nezumiiro & hai /guree & hai \\
BLACK & kuro & kuro & kuro \\
BROWN & cha(iro) & cha & cha \\
RED & aka(iro) & aka & aka \\
ORANGE & daidai(iro) & daidai / orenji & orenji \\
YELLOW & ki(iro) & ki & ki \\
GREEN & midori(iro) & midori & midori \\
LIGHT BLUE & - & mizu (?) & mizu \\
BLUE & ao & ao & ao \\
PURPLE & murasaki(iro) & murasaki & murasaki \\
PINK & momoiro & momo/pinku & pinku \\
\hline
\end{tabular}

Note:

B\&K = Berlin \& Kay (1969), U\&B = Uchikawa \& Boynton (1987), K et al. = Kuriki et al. (2017). 
Standard Japanese has (at least) the 11 basic colour terms set out by Berlin \& Kay (1969). The term mizu(iro) for 'light blue' has been suggested as a twelfth term based on both its frequent and consistent use across speakers (Uchikawa \& Boynton 1987; Kuriki et al. 2017), and on linguistic grounds (Stanlaw 1987; Conlan 2005). Secondly, the findings in both Uchikawa \& Boynton (1987) and Kuriki et al. (2017) seem to indicate that the term nezumi(iro) for GREY has fallen out of use since the original study by Berlin \& Kay (1969). Finally, the effects of internationalisation are clearly noticeable, as the native words momo(iro) 'pink' and daidai(iro) 'orange' have largely been replaced by their English loanword counterparts pinku and orenji ${ }^{1}$ (Uchikawa \& Boynton 1987; Kuriki et al. 2017). Uchikawa \& Boynton (1987) reported a similar tendency for the term hai(iro) 'grey', however Kuriki et al. (2017) showed that this replacement did not occur. It is important to note here that the large number of basic colour categories already reported in Japanese by Berlin \& Kay (1969) mean that the changes reported in later studies mainly relate to the colour terms used to describe those categories. While there has been no direct comparison between the colour system of Japanese at different points in time, the addition of 'light blue' as basic category, rather than a minor category, appears to be the most significant semantic change, and mirrors the distinction in other languages, such as Turkish (Özgen \& Davies 1998), Russian (Paramei 2005) and Greek (Athanasopoulos 2009).

While the contemporary colour system of Japanese conforms to the Berlin and Kay framework, its developmental trajectory shows a few anomalies. The long history of writing in Japan has made it possible to track changes in the colour lexicon (Stanlaw 2007; Stanlaw 2010). Stanlaw (2007, 2010) theorises about earlier stages of colour terminology based on linguistic and cultural data - see Table 2 for an overview.

At the time of the earliest writings in the 8th century, there were eight colour terms in use. Strikingly, 'purple' appears early in this data, which is crosslinguistically uncommon and not predicted by the original Berlin \& Kay framework. However, Stanlaw (2007) argues there is strong evidence for purple being a culturally salient colour. The limited availability of purple dye, and its resulting exclusivity, has made the colour purple important in many cultures, often linked to high status and power (Hendrick-Wong 2013). While the exact timing of the divergence between Japanese and Ryukyuan is still under debate, most theories

1. The fact that 'orange' as a colour category was not established until the Meiji Era (1868-1912) - a period with significant Western influence - combined with the slightly earlier appearance of orenjiiro (1897 1897, cf. daidaiiro 1905; Shogakukan 2000) could raise some questions about whether the native term daidai(iro) ever, in fact, achieved basic colour term status. 
Table 2. Colour terms added in first four steps of the evolution of the Japanese colour lexicon, with approximate dates for each stage (Stanlaw 2007)

\begin{tabular}{|c|c|c|c|}
\hline $\begin{array}{l}\text { I } \\
400 \mathrm{CE}\end{array}$ & $\begin{array}{l}\text { II } \\
500 \mathrm{CE}\end{array}$ & $\begin{array}{l}\text { III } \\
650 \mathrm{CE}\end{array}$ & $\begin{array}{l}\text { IV } \\
750 \mathrm{CE}\end{array}$ \\
\hline $\begin{array}{l}\text { aka 'red' } \\
a o \text { 'grue' }\end{array}$ & $k i$ 'yellow' & murasaki 'purple' & $\begin{array}{l}\text { midori 'green' } \\
\text { momo 'pink' }\end{array}$ \\
\hline $\begin{array}{l}\text { kuro 'black' } \\
\text { shiro 'white' }\end{array}$ & & & \\
\hline
\end{tabular}

Note:

Stage IV represents that of the first writing, i.e. there were eight terms.

place it well before the 8th century (Pellard 2015, for an overview), meaning that the Japanese and Ryukyuan colour lexicons developed independently from an ancestral language with six or fewer colour terms.

For some initial insights into the colour lexicon of the Ryukyuan languages, we can turn to dialect dictionaries such as the Dictionary of contemporary Japanese dialects (Hirayama 1992), which includes data for several Ryukyuan varieties and has entries for seven colour terms: aka 'red, a warm color'; kiiro 'yellow color'; midori 'green, verdure'; ao 'blue, sky blue, azure, indigo blue, green'; murasaki 'purple, amethyst'; kuro 'black color', and shiro 'white' (translations as found in the dictionary). Table 3 is a summary of entries for the five chromatic colour terms in Standard Japanese and the Ryukyuan varieties.

Table 3. Entries for the five chromatic colours in the Dictionary of contemporary Japanese dialects (Hirayama 1992) for standard Japanese and four Ryukyuan languages

\begin{tabular}{clllll}
\hline & RED & YELLOW & GREEN & BLUE & PURPLE \\
\hline Standard & aka & ki:ro & midori & ao & murasaki \\
Ryukyuan & & & & & \\
Amami & ha: & $k^{\text {?}}$ i?iru & midori & Po?iro & murasaki \\
Okinawa & ?aka: & ki:ru: & Po:ru: & Po:ru: & Po:ru: \\
Miyako & aka & $k^{s i}: r u \sim t s i: r u^{*}$ & o: au & o: au & murasaki* \\
Yaeyama & Paga & ki:ru & Pau & Pau & murasaki* \\
\hline
\end{tabular}

Note:

Asterisked entries are specifically mentioned to be relatively new expressions resulting from standard language influence. 
The table shows that a distinction between 'green' and 'blue' is not generally made in the Ryukyuan languages. Furthermore, it seems that Okinawa extends the use of Po:ru: to include 'purple', whereas the other languages distinguish it as a separate category. However, the Miyako and Yaeyama entries specifically mention that the term murasaki for purple is a relatively new expression that has been introduced through contact with the standard language, which is also suggested by resemblance to the Standard Japanese form. Finally, some entries for the 'yellow' term in Miyako also mention standard language influence. This colour system with fewer distinctions is expected if we assume that Ryukyuan indeed split from Japanese before the 8th century.

Aside from information in dictionaries, there is one large-scale study that uses colour stimuli in a naming task. Kusakabe (1964) collected naming data for 15 chromatic colour cards from 89 speakers across 68 locations in Okinawa Prefecture. ${ }^{2}$ The study showed that Ryukyuan speakers used between two and four chromatic colour terms, largely cognate with the oldest colour terms in Japanese - aka 'red', ao 'grue', ki 'yellow' and murasaki 'purple' (Stanlaw 2007, 2010). The Miyako and Yaeyama 'purple' terms in Kusakabe (1964) were listed as having the form mura $(t) s i k i,{ }^{3}$ which differs from the form murasaki in Table 3 . The note in Hirayama (1992) that murasaki is relatively new thus likely comments on the specific form rather than the colour category as such. Unfortunately, there has been no systematic study of Ryukyuan colour terminology since, leaving it unclear what changes have occurred more recently.

To summarise, while the Standard Japanese colour lexicon has been studied since the 1960 s, the most extensive inquiry into the colour lexicon of the related Ryukyuan languages is more than 50 years old. Given the developments in the Japanese colour lexicon during that time, as well the changing linguistic landscape across the Ryukyu Islands described above, the question arises what impact this has had on the colour lexicon of contemporary Ryukyuan speakers. Has the diminished use of Ryukyuan led to an increase of Standard Japanese colour terms by Ryukyuan speakers and if so, do these include the English loanwords that have gained widespread use in Standard Japanese in recent decades?

\subsection{The current study}

The current study aims to gain insight into the modern colour lexicon of Ryukyuan and assess the influence from Standard Japanese and English. In the

2. The study includes data from four Ryukyuan languages (Okinawa, Miyako, Yaeyama and Yonaguni), but excludes Amami Ryukyuan, which is spoken in Kagoshima Prefecture.

3. Miyara (1930) also lists the similar form muraçiki for Taketomi dialect (Yaeyama). 
first part, we describe how colour naming data was collected from modern Ryukyuan speakers, and outline the colour terms and categories in the languages under consideration, focusing on potential outside influence - i.e., Standard Japanese or English. We then explore semantic variation in a core set of colour terms - aka 'red', $k i$ 'yellow', ao 'grue', and murasaki 'purple' - which were chosen because of their longevity (Stanlaw 2007, 2010) and their use in both Japanese (Berlin \& Kay 1969; Uchikawa \& Boynton 1987; Kuriki et al. 2017) and Ryukyuan (Kusakabe 1964; see also Hirayama 1992). We compare the semantic distances between these colour categories using a statistical procedure applied by Jäger (2012). Finally, we investigate the semantic changes that have occurred in Ryukyuan over time by comparing the newly collected naming data to earlier data from Kusakabe (1964).

\section{Methods}

\subsection{Languages and speakers}

Data was collected from speakers in three Ryukyuan language areas as well as from Standard Japanese for comparison - see Figure 1 - during four fieldtrips conducted between 2017 and 2019. An attempt was also made for data collection with Okinawan with two speakers in a session; but this was stopped at the request of speakers after they were unable to name several colour chips. As there is no standardised variety of any of the Ryukyuan languages (Heinrich et al. 2015), data was collected from multiple localities, i.e., in multiple dialects. We will use the term "language area" when describing Ryukyuan for the remainder of this paper, e.g., the Amami language area, so we can refer to each (mutually unintelligible) Ryukyuan language without having one specific variety as its standard. As the Ryukyuan languages are endangered, data was collected from elderly native speakers; we also collected data from older speakers in Standard Japanese to provide a comparable sample. The data presented here also constitutes the first systematic investigation of colour naming in the Amami language area, since it was not featured in the Kusakabe (1964) study.

\subsection{Materials and procedure}

Some of the elderly speakers tested had little experience in performing abstract, reflective language tasks and, therefore, some naming sessions were conducted with multiple speakers simultaneously. As a result, all analyses were performed on sessions rather than speakers - see Section 2.3. Speakers were tested indoors in 


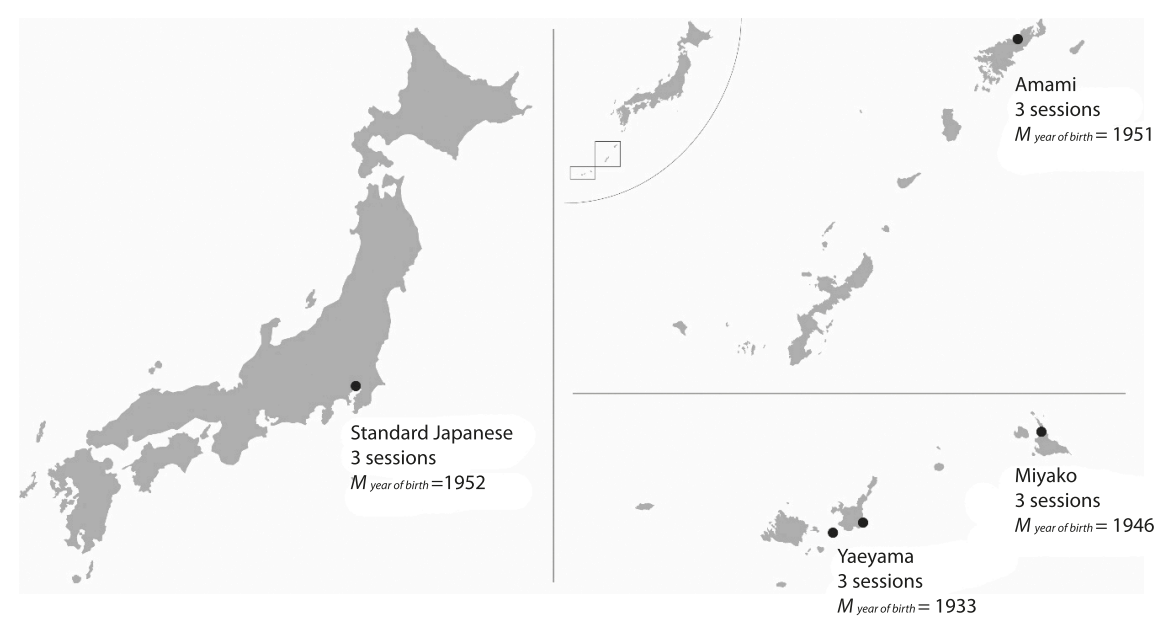

Figure 1. Map of Japan (left) and the Ryukyu islands (right) with fieldwork locations marked, as well as session and participant information

natural lighting conditions. Speakers were pre-tested for their colour vision using an Ishihara test, and we only conducted further testing with speakers that passed it. For the colour naming task, we used a set of 84 Munsell colour chips (Majid et al. 2011) - see Figure 2. ${ }^{4}$ The colour chips were presented one by one, in two fixed orders - one being the reverse of the other - after which speakers were asked to freely name the colour of the chip in either Standard Japanese or their local Ryukyuan variety, as appropriate in each site. Speakers were allowed to give multiple responses of any length. Sessions were audio (and sometimes video) recorded for later transcription. Speakers gave informed consent before participating, and all data was collected under the Ethics Assessment Committee of the Centre for Language Studies at Radboud University.

\subsection{Coding}

All full responses were transcribed. Colour terms can appear in several forms in Japanese and Ryukyuan varieties. Moreover, as speakers were told to freely describe the colour chips, they used a range of different naming strategies - see Examples 1 through 4 .

4. For the full colour figure, please refer to the online version of this article. 
(1) STANDARD JAPANESE CODED RESPONSE:

$\underline{a o}=n i \quad$ chikai $\rightarrow \underline{a o}$

$\underline{\text { blue }}=$ DAT near:NPST

'(it is) close to blue.'

(2) AMAMi RYUkyUan (TATSUgo) CODED Response:

$\underline{0: s a n} \rightarrow \underline{0:}$

blue:NPST

'(it is) blue.'

(3) MIYAKO RYUKYUAN (NISHIHARA) CODED RESPONSE:

$\underline{a u-m u n u} \rightarrow \underline{a u}$

blue-thing

'blue.'

(4) YAEYAMA RYUKYUAN (SHIRAHO) CODED RESPONSE:

$\underline{0:-O:-s e r o} \rightarrow \underline{0:}$

blue-blue-do:CONT

'(it is) blue.'

In addition to the full responses we extracted, per session, the main response(s) for each colour chip. We defined the main response as either the root of the basic colour term, or the source term that is the core descriptor for each colour chip. All nominal, adjectival and verbal-suffixing, as well as reduplications and modifier phrases were excluded - see Coded response on right-hand side of Examples 1 through 4 .

\section{Results}

We first provide a general overview of the colour terms used by contemporary speakers of the Ryukyuan languages and assess the influence from Standard Japanese and English in Section 3.1. We then focus on the colour categories distinguished by contemporary speakers of the Ryukyuan languages in Section 3.2. Next, we present an analysis of the semantic variation across a core set of colour terms: aka 'red', ao 'grue', $k i$ 'yellow' and murasaki 'purple' in Section 3.3. Finally, Section 3.4 compares our newly collected data to the findings previously reported in Kusakabe (1964). 


\subsection{Colour terms in contemporary Ryukyuan and Japanese}

We used the frequencies of coded responses to get a general overview of the colour terms elicited in the naming task. As the languages under investigation in this study are related, we were interested in establishing the extent to which each term is used across the language family. In addition, such an overview provides some initial clues about the extent of outside influences such as Standard Japanese terms and English loanwords. Table 4 shows, per language area, the responses that occurred at least three times and that were used in more than one session. Each row in the table contains one cognate set, with rows arranged by an approximate colour gradient. We grouped together four types of responses: (1) abstract colour terms that denote a colour quality without referring to a concrete source object (e.g., banana, sky), (2) terms that have been designated as basic colour terms in Standard Japanese, but that are or were originally source-based, (3) source-based terms that are generally not considered basic colour terms, and (4) English loanwords.

The Standard Japanese data largely recapitulates previous naming studies (Berlin \& Kay 1969; Uchikawa \& Boynton 1987; Kuriki et al. 2017; see Table 1). The native word momo(iro) 'pink' $(n=19)$ outnumbered the English loanword pinku $(n=6)$ and all speakers used daidai(iro) instead of orenji. The relatively large number of native colour terms used for 'orange' and 'pink', as opposed to their English loanword counterparts, recapitulate the findings reported by Uchikawa \& Boynton (1987) more than Kuriki et al. (2017). This is most likely because the sample of speakers, who were born in the 1940s-1950s, are demographically most similar to Uchikawa \& Boynton (1987). Nevertheless, the term mizu(iro) was also frequently used, indicating the emerging salience of 'light blue' as a category as reported in Kuriki et al. (2017).

Overall, the total the number of terms elicited in each of the four language areas was comparable, although the number of colour terms produced per individual session ranged between 13 and 22, with the exception of one Miyako speaker who used only 4 terms, specifically mentioning that "there are only four colours in Miyako". Several other Ryukyuan speakers commented that their language variety "does not have many words for colours", also stating more specifically that "there are no separate words for blue and green", although they did appeal to Standard Japanese colour terms, English loanwords or source-based descriptions to make finer distinctions on occasion. Overall, most speakers demonstrated at least some meta-linguistic awareness of their bilingualism, as well as differences between the colour lexicons of both languages.

Most of terms used across the Ryukyuan languages have cognates in Standard Japanese. The six oldest colour terms aka, ao, kuro, shiro, ki and murasaki 
Table 4. Responses produced at least three times in more than one session in the naming task

\begin{tabular}{lllll}
\hline \multicolumn{2}{l}{ Abstract colour terms } \\
\hline Japanese & Amami & Miyako & Yaeyama & Gloss \\
\hline shiro & shiro & $s s u$ & $s s u$ & 'white' \\
kuro & $k u r o$ & $f f u$ & $f f u$ & 'black' \\
aka & $h a:$ & $a k a$ & $a g a$ & 'red' \\
$k i$ & $k i:$ & $k i:$ & $k i N$ & 'yellow' \\
ao & $o:$ & $a u$ & $o:$ & 'blue' \\
& & $k o n$ & & 'dark blue'
\end{tabular}

Historically source-based terms designated as basic colour terms in Standard Japanese

\begin{tabular}{|c|c|c|c|c|c|}
\hline Japanese & Amami & Miyako & Yaeyama & Gloss & \\
\hline & & & & Colour & Source \\
\hline \multirow[t]{2}{*}{ hai } & hai & & hai & 'grey' & 'ash' \\
\hline & & nezumi & & 'grey' & 'mouse' \\
\hline \multirow{6}{*}{$\begin{array}{l}\text { cha } \\
\text { daidai } \\
\text { midori } \\
\text { mizu } \\
\text { murasaki } \\
\text { momo }\end{array}$} & cha & & cha & 'brown’ & 'tea' \\
\hline & daidai & daidai & daidai & 'orange' & 'C. $\times$ daidai citrus fruit' \\
\hline & midori & midori & midori & 'green’ & 'verdure' \\
\hline & mizu & & & 'light blue' & 'water' \\
\hline & murasaki & murasaki & murasaki & 'purple' & 'L. erythrorhizon plant' \\
\hline & momo & & & 'pink' & 'peach' \\
\hline \multicolumn{6}{|c|}{ Source-based terms } \\
\hline \multirow[t]{2}{*}{ Japanese } & Amami & Miyako & Yaeyama & \multicolumn{2}{|l|}{ Gloss } \\
\hline & & $a z u k i$ & & \multicolumn{2}{|c|}{ 'adzuki bean (V. angularis)' } \\
\hline \multirow[t]{2}{*}{ hada } & & & & \multicolumn{2}{|l|}{ 'skin’ } \\
\hline & & & $k a: r a$ & \multicolumn{2}{|l|}{ 'roof tile' } \\
\hline tsuchi & tsuchi & tsuchi & & \multicolumn{2}{|l|}{ 'soil' } \\
\hline \multirow[t]{2}{*}{ oudo } & oudo & & & \multicolumn{2}{|l|}{ 'yellow soil' } \\
\hline & & & fumbutu & \multicolumn{2}{|c|}{ 'fukugi tree (G. subelliptica)' } \\
\hline \multirow[t]{2}{*}{ kusa } & & & ssa & \multicolumn{2}{|l|}{ 'grass' } \\
\hline & & & pa: & \multicolumn{2}{|l|}{ 'leaf' } \\
\hline \multirow[t]{3}{*}{ sora } & sora & sora & & \multicolumn{2}{|l|}{ 'sky' } \\
\hline & & & $\operatorname{tin}$ & \multicolumn{2}{|l|}{ 'sky’ } \\
\hline & & & tu: & \multicolumn{2}{|l|}{ 'sea' } \\
\hline \multicolumn{3}{|l|}{$a i$} & & \multirow{2}{*}{\multicolumn{2}{|c|}{ 'indigo plant (P. tinctorial)' }} \\
\hline English loa & nwords & & & & \\
\hline
\end{tabular}


Table 4. (continued)

\begin{tabular}{lllll}
\hline \multicolumn{2}{l}{ Abstract colour terms } & & & \\
\hline Japanese & Amami & Miyako & Yaeyama & Gloss \\
\hline \multirow{2}{*}{ guriin } & orenji & orenji & 'orange' \\
& & burui & guriin & 'green' \\
& & 'blue' \\
pinku & pinku & pinku & pinku & 'pink' \\
\hline
\end{tabular}

Note:

As the naming data was collected from several dialects, the responses listed in the table list the most frequent form in each language area.

(Stanlaw 2007, 2010) were used in all sessions. Of these, the four oldest ( $a k a$, ao, kuro and shiro) were virtually always used in their local pronunciations - see Table 4. The 'yellow' term $k i$ had a mixture of local and standard-like pronunciations, but the 'purple' term murasaki was most often in Standard Japanese pronunciation, in contrast to the reported mura(t)siki in Kusakabe (1964). Several other terms were produced in Standard Japanese forms, such as momo(iro) 'pink; lit. peach' where mumu was expected, and tsuchi(iro) 'lit. soil (colour)', sora(iro) 'lit. sky (colour)' by Miyako speakers even though the local word for 'soil' is $m t a \sim n t a$ and 'sky' is tin. For the Yaeyama language area, we find tin nu iru '[sky GEN colour]' The strongest influence of Standard Japanese on Ryukyuan is found in the high frequency of midori 'green' across all Ryukyuan sessions, which as we discuss in Section 3.4 was not reported in the Ryukyuan data of Kusakabe (1964). In addition, the form of this colour term was predominantly midori as in Standard Japanese. If the term had been integrated into the Ryukyuan languages, the various sound changes across the languages would have produced miduri, midui, midurï or midul, but these were not attested in the naming data.

Ryukyuan speakers used several English loanwords, of which pinku was the most frequent. Even though orenji has been shown to have all but replaced the native term daidai(iro) in Standard Japanese across several studies (e.g., Kuriki et al. 2017), Ryukyuan speakers did not use orenji to a comparable level. Given their prominence in Standard Japanese in recent decades, it is difficult to directly determine whether the use of these English loanwords by Ryukyuan speakers is an influence from the standard language or from English. However, a potential Ryukyuan-specific development is the relatively frequent use of English guriin to distinguish green parts of the spectrum - no Standard Japanese speakers used an English loanword to do so.

Speakers from all language areas used source-based terms, many of which have been previously reported in Standard Japanese (e.g., kusa 'grass' and sora 
'sky', see Kuriki et al., 2017). However, the Ryukyuan sessions produced a few that were specific to the local area such as ka:ra referring to the orange/brown roof tiles found on Taketomi Island, or fumbutu referring to the G. subelliptica tree species commonly found around the Ryukyu Islands, which is used for yellow dye. Yaeyama speakers in particular used relatively more source-based terms and interestingly, these were again used for green parts of the spectrum as with the Standard Japanese terms and English loanwords. Green shades were described with the source-based terms ssa 'grass' (< Standard Japanese kusa), pa: 'leaf/ leaves' (< Standard Japanese $h a$ ), or a combination of the two (ssa nu pa: '[grass GEN leaves]').

\subsection{Colour categories in contemporary Ryukyuan and Japanese}

We used the modal (i.e., most frequent) responses per colour chip to uncover the most salient colour categories in each language area. Figure 2 (top panel) shows the 84 colour chips arranged by hue (coded o through 20) and brightness (coded A through D). The panels below show the modal response for each colour chip in each of the four language areas. Where speakers specifically commented that terms were synonyms, e.g., midori, guriin and ssa for 'green' in Yaeyama, we counted these together for the purpose of these figures, but elsewhere keep them distinct. We then coloured all chips with the same dominant response the same colour. Blank chips are those for which there was no modal response, i.e., there was no single dominant term across sessions.

The Japanese data is consistent with what has been reported previously: the eleven basic colours identified by Berlin \& Kay (1969) - 'white', 'grey', 'black', 'brown', 'red', 'orange', 'yellow', 'green', 'blue, 'purple', and 'pink' - were clearly distinguished. In addition, a 'light blue' category emerged as suggested by later scholars (Uchikawa \& Boynton 1987; Kuriki et al. 2017), as well as 'peach' and 'light brown', consistent with Kuriki et al. (2017). So full coverage of the color space in Standard Japanese was achieved through 14 distinct terms.

Fewer categories emerged in the Ryukyuan language areas, with 13 colours in Amami, 12 in Miyako and 11 in Yaeyama. Overall, the Ryukyuan languages areas and Standard Japanese showed evidence of the eleven basic colours originally suggested by Berlin \& Kay (1969). In addition, the 'light blue' category emerged in Japanese, Amami and Miyako language areas but not in Yaeyama; and 'light brown' was evident in Amami, but not in the other two language areas. There was no evidence of a 'peach' category in Ryukyuan.

Figure 2 also illustrates a number of differences between Japanese and Ryukyuan in the 'grue' area. Japanese neatly divides 'green' and 'blue', but this division is not established across the Ryukyuan languages where the two categories 

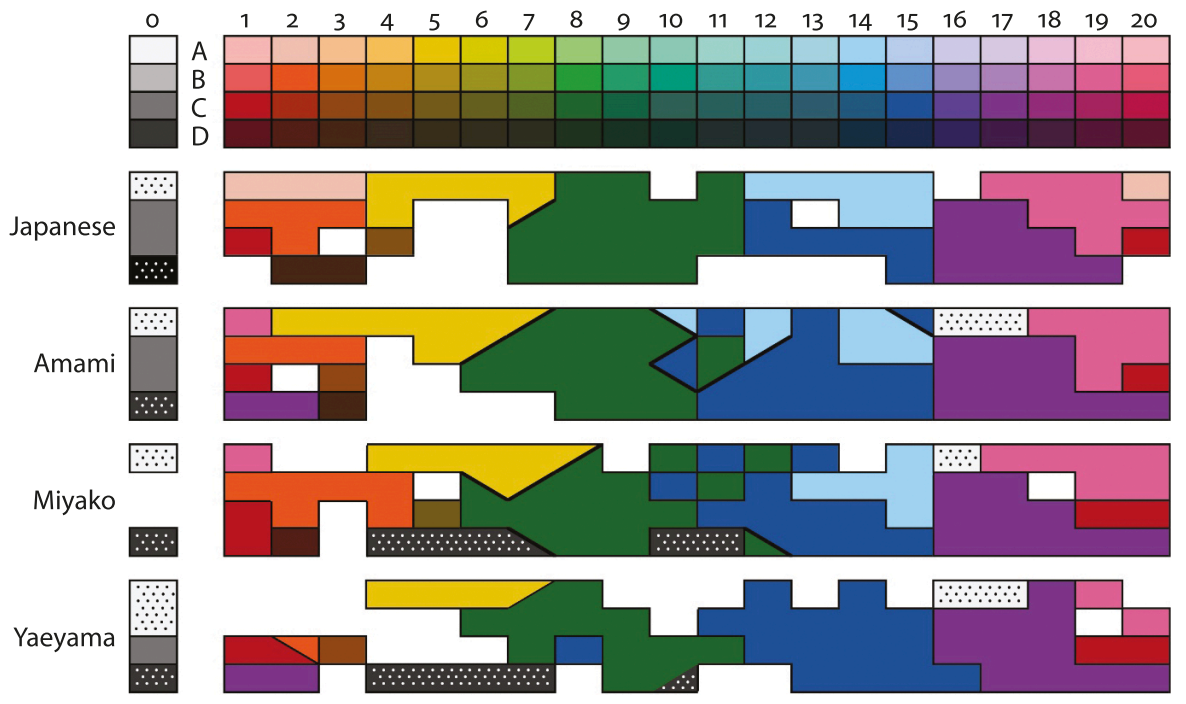

Figure 2. Modal responses per language for the 84 colour chips. Top grid presents an approximation of the colour chips used for elicitation

seem to overlap and intersect in the boundary area. In addition, while 'light blue' was present in Amami and Miyako, it is not as clearly defined as in Standard Japanese, Also, whereas murasaki is only used for the 'purple' part of the spectrum in Japanese, the Ryukyuan languages seem to extend its range to include darker shades of 'red'. Finally, Standard Japanese shiro and kuro are limited to achromatic colours ('white' and 'black', respectively), whereas the three Ryukyan language areas extend the use of these terms to include lighter and darker shades in general (see chips in rows A and D respectively), suggesting they are being used with the meanings 'dark' and 'light'. This usage resembles that of the oldest stages of the Japanese colour system (Stanlaw 2007, 2010).

In addition, Figure 2 hints at further differences between the three Ryukyuan language areas. In contrast to Amami and Miyako speakers, Yaeyama speakers did not distinguish shades of blue. Furthermore, while the Amami 'white' term was used to include lighter shades in general, the 'black' term was not used for general dark shades in contrast to Miyako and Yaeyama. Finally, 'orange' and 'pink' did not emerge as prominently in Yaeyama as in the other language areas.

To summarise, while the modal responses from the naming task indicate that Ryukyuan varieties have fewer colour categories, several categories not previously reported emerged, e.g., 'green', 'orange' - cf. Kusakabe (1964). However, the boundaries of these new categories appear to be less clearly defined than in Japanese. 


\subsection{Semantic variation in a core set of colour terms}

We next zoom in on the semantic variation across a core set of colour vocabulary. We looked specifically at the extensional range of the cognate sets $a k a, a o, k i$ and murasaki across the stimulus set, which were chosen because of their longevity and their widespread use in the language family. To statistically compare the cognate sets across the language areas, we performed principal component analysis (PCA) to uncover the units that formed the basis for these categories, using the approach set out by Jäger (2012).

To minimise the effect of idiosyncratic responses, we only included colour terms that were used in more than one session and were used more than three times - i.e., terms in Table 4. Following Jäger (2012), we created a colour term * colour chip matrix for each session, in which we coded the frequencies of the terms used for each chip, and then summed the matrices for all sessions per language area. Next, each row was divided by the number of sessions in which the term was used, after which each row was copied as many times as the response occurred to give more weight to more frequent responses. Finally, we stacked the four language area matrices, resulting in a $190 \times 84$ contingency table that was used for the principal component analysis.

We used the scree test (Cattell 1966) to determine the number of principal components and continued with a solution of 12 components accounting for $75.5 \%$ of the variance. ${ }^{5}$ Ten of the eleven basic colour categories proposed by Berlin and Kay (1969) were represented as principal components, with only 'grey' missing. We rotated the PCA solution using Varimax rotation to maximise the components. As principal component analysis separates statistically important variation from noise, we used the 12 extracted components to project the four chromatic cognate sets ( $a k a, a o, k i$, murasaki) back onto the colour chip array (see Jäger 2012:526-531). The PCA solution was used to calculate cosine similarities between each set of colour terms as a measure of similarity between languages, which were then used in separate cluster analyses for each cognate set, as well as an aggregate cluster analysis.

5. Jäger (2012) used the Kaiser criterion (all factors with an eigenvalue $>1$; Kaiser 1960) to select the number of principal components, which in our cases would mean selecting 17 principal components, together accounting for $82.8 \%$ of the variance in the data. However, inspection of this solution showed several components that were hard to interpret - especially in light of the naming data presented in Sections 3.1 and 3.2. 
3.3.1 The $a k a$ ' (macro) red' cognate set

Figure $3 a$ shows the range of the aka cognate set across the four languages, Figure $3 \mathrm{~b}$ shows the cosine similarities, and Figure $3 \mathrm{c}$ is a plot of the cluster analysis based on these cosine similarities. The cluster analysis revealed Miyako to be the most distinct, and that Amami and Yaeyama were more similar to each other than they were to Japanese. As the figure shows, speakers of Ryukyuan varieties extend the use of $a k a$ further into the orange part of the spectrum, whereas Japanese speakers restrict its use to a small set of red chips. Miyako is the only variety where $a k a$ is extended to include yellow hues too.

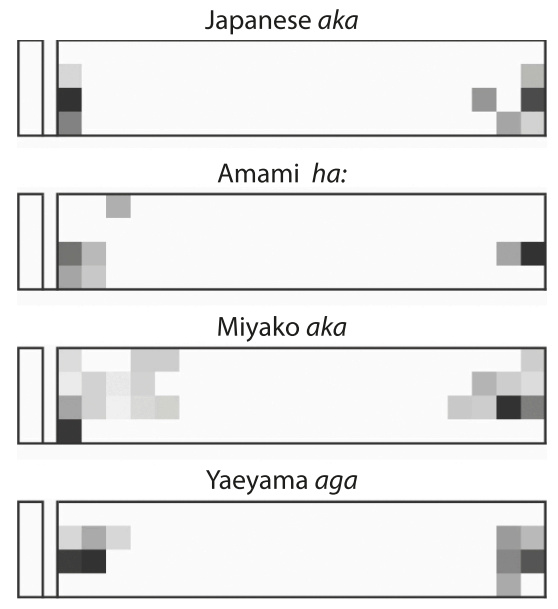

(a)

\begin{tabular}{lccc}
\multicolumn{4}{l}{ Cosine similarities for the aka cognate set } \\
\hline & Amami & Miyako & Yaeyama \\
\cline { 2 - 4 } Japanese & 0.736 & 0.806 & 0.627 \\
Amami & & 0.917 & 0.860 \\
Miyako & & & 0.850 \\
\hline
\end{tabular}

(b)

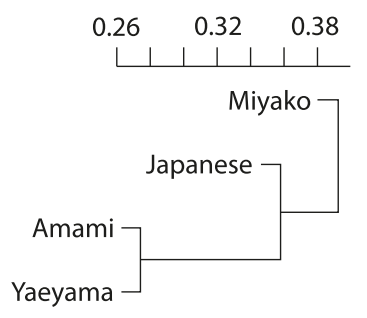

(c)

Figure 3. (a) The range of the aka cognate across the four language areas, (b) cosine similarities calculated based on principal component analysis, and (c) cluster analysis results based on these cosine similarities

\subsubsection{The $k i$ 'yellow' cognate set}

As with $a k a$, the cluster analysis revealed Miyako to be the most distinct, with Amami and Yaeyama more similar to each other than to Japanese. Figure 4a shows that speakers of Ryukuan tend to extend the use of $k i$ further into orange and green, as well as for darker shades than Japanese speakers.

\subsubsection{The ao 'grue/blue' cognate set}

The cluster analysis in Figure $5 \mathrm{C}$ reveals the three Ryukyuan language areas were more similar to each other than they were to Japanese. Speakers of the Ryukyuan varieties tended to extend the use of ao further into green, whereas Japanese speakers restricted its use to blue. Within Ryukyuan, Amami and Miyako speakers 


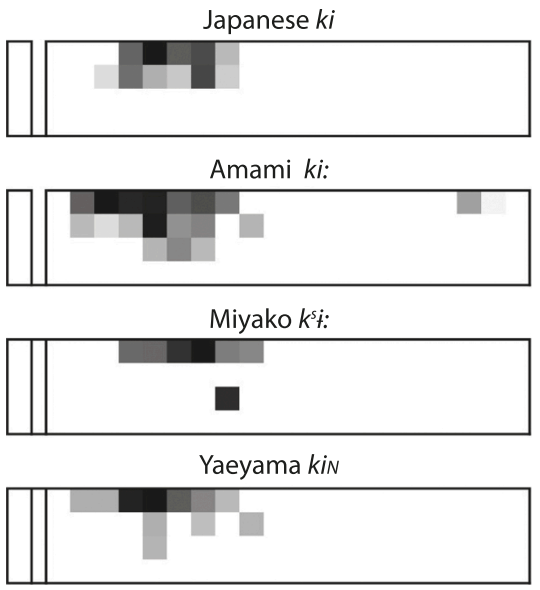

(a)
Cosine similarities for the ki cognate set

\begin{tabular}{lccc}
\hline & Amami & Miyako & Yaeyama \\
\cline { 2 - 4 } Japanese & 0.750 & 0.666 & 0.812 \\
Amami & & 0.549 & 0.856 \\
Miyako & & & 0.691 \\
\hline
\end{tabular}

(b)

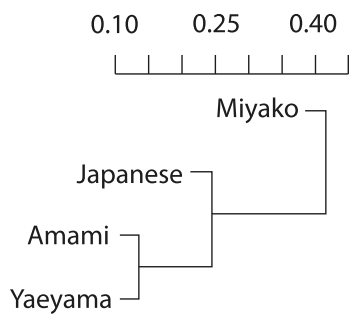

(c)

Figure 4. (a) The range of the ki cognate across the four language areas, (b) cosine similarities calculated based on principal component analysis, and (c) cluster analysis results based on these cosine similarities

tended to have larger green categories than Yaeyama speakers, as seen in Figure $5 \mathrm{a}$ which depicts the range of the ao cognate set across the four languages.
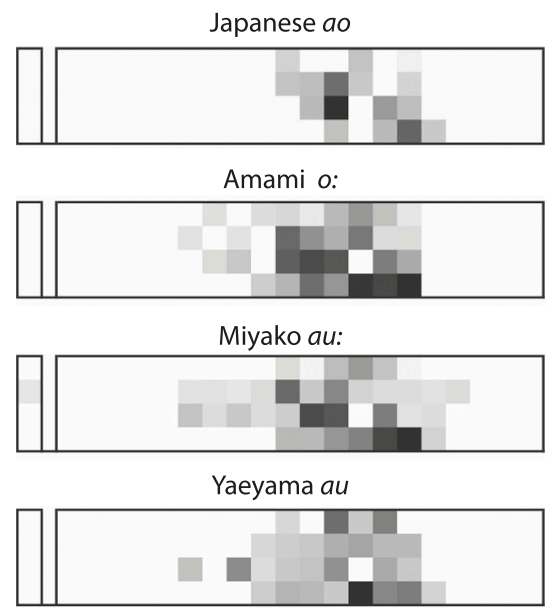

(a)

\begin{tabular}{lccc}
\multicolumn{4}{l}{ Cosine similarities for the ao cognate set } \\
\hline & Amami & Miyako & Yaeyama \\
\cline { 2 - 4 } Japanese & 0.717 & 0.596 & 0.613 \\
Amami & & 0.701 & 0.725 \\
Miyako & & & 0.606
\end{tabular}

(b)

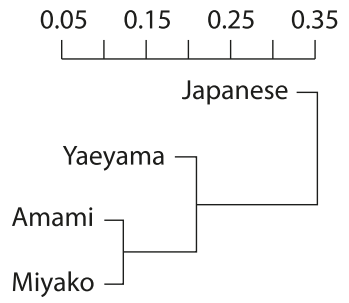

(c)

Figure 5. (a) The range of the ao cognate across the four language areas, (b) cosine similarities calculated based on principal component analysis, and (c) cluster analysis results based on these cosine similarities 


\subsubsection{The murasaki 'purple' cognate set}

As with $a o$, the cluster analysis revealed the three Ryukyuan varieties were more similar to each other than they were to Japanese. Figure 5a shows that speakers of Ryukyuan tended to use murasaki to include dark shades of red, whereas Japanese speakers tended to limit the use to chips between blue and red. As another point of contrast, Ryukyuan speakers limited the use of murasaki to darker colour chips, whereas Japanese murasaki also included lighter chips.

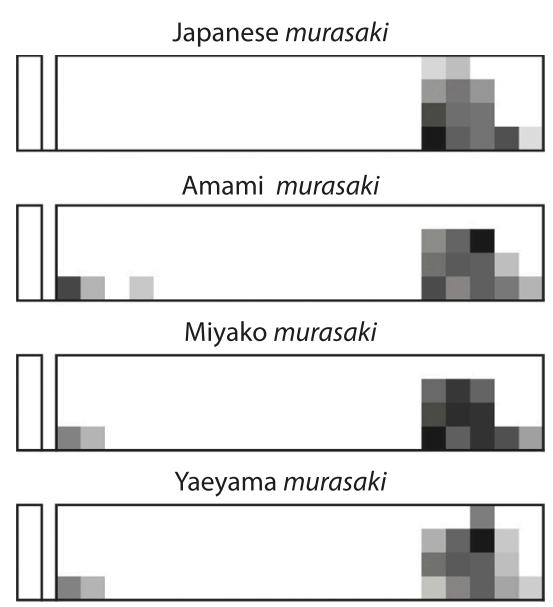

(a)

\begin{tabular}{lccc}
\multicolumn{3}{c}{ Cosine similarities for the murasaki cognate set } \\
\hline & Amami & Miyako & Yaeyama \\
\cline { 2 - 4 } Japanese & 0.858 & 0.941 & 0.781 \\
Amami & & 0.954 & 0.926 \\
Miyako & & & 0.885 \\
\hline
\end{tabular}

(b)

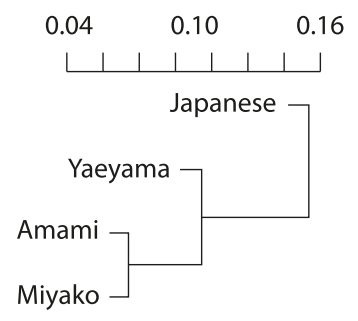

(c)

Figure 6. (a) The range of the murasaki cognate across the four language areas, (b) cosine similarities calculated based on principal component analysis, and (c) cluster analysis results based on these cosine similarities

\subsubsection{Overall cluster analysis for the cognate sets}

Finally, we computed an aggregate similarity measure between the four language areas by calculating the mean of the cosine similarities for the four colour terms, and performed a cluster analysis using this aggregate similarity measure (Figure 7). This overall cluster analysis confirmed that the Ryukyuan languages are distinct from Japanese. Within the Ryukyuan languages, Miyako appears the most distinct.

To summarise, a comparison of four colour cognate sets ( $a k a, k i$, ao and murasaki) using principal component analysis showed that the Ryukyuan languages are always distinct from Japanese. The analysis also revealed that the languages group together differently for each cognate set, which suggests the extensional ranges of colour terms develop along individual trajectories in each language. 


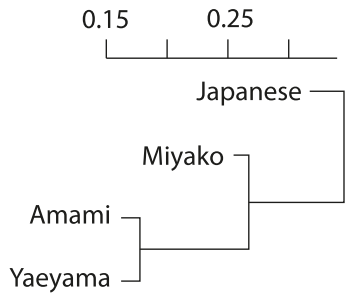

Figure 7. Cluster analysis based on the mean cosine similarity for the four cognate sets (aka, ki, ao and murasaki) taken together

\subsection{Semantic change in the Ryukyuan colour lexicon}

As mentioned in the introduction, the only systematic study of colour naming across the Ryukyus is Kusakabe (1964). We compared our data to this older Ryukyuan data in order to put our new naming data into further historical context and study semantic changes in the extensional use of the Ryukyuan colour lexicon. Since the two studies used different methods, we reanalysed the Kusakabe data for comparability. Kusakabe (1964:62) used 15 colour cards from the Japan Colour Research Institute, which he labelled $a$ through $O{ }^{6}$ We determined the closest approximations of these colour cards in our sample of 84 colour chips, shown in Figure 8. Kusakabe's colour cards $g, M, N$ and $O$ fall halfway between two of our colour chips. Colour cards $a$ and $E$ are not represented because these are distinctions not captured in our sample - in the red and yellow parts of the spectrum, respectively.

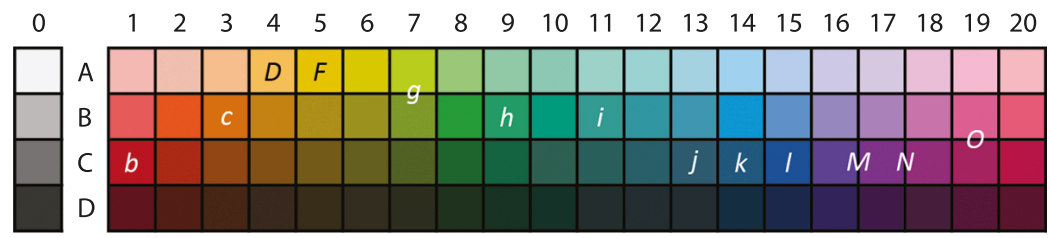

Figure 8. Overview of the 84 Munsell colour chips used in the current study, with closest approximations in the Kusakabe (1964) study marked

Kusakabe (1964) focused on four chromatic colour term cognates: aka 'red', ao 'grue/blue', $k i$ 'yellow', and murasaki 'purple'. As a result, his coding scheme

6. The author labelled the colour cards using both lowercase and uppercase (abcDEFghijlklMNO) as part of his coding scheme representing their (idealised) naming in Standard Japanese. We follow these original labels. 
only codes these four terms and all other responses were coded as 'other.7 For a direct comparison, we therefore recoded responses from our naming sessions following Kusakabe's conventions, i.e. only counting (cognate forms of) aka, ki, ao and murasaki, and coding all remaining responses as other. We created five new matrices in which we coded how many times each response (aka, ki, ao, murasaki, other) was used for each of the 13 colour chips shared between the current study and Kusakabe (1964). Two matrices contained Miyako data (1964; current), two contained Yaeyama data (1964; current), and one contained Standard Japanese data. ${ }^{8}$ We do not consider the Okinawan data from Kusakabe (1964), as we were not able to elicit Okinawan data from speakers. As mentioned earlier, data collection was attempted with this variety, but was not possible.

The chip-by-response matrices were used to calculate distances between the five language areas, past and present. We chose chi-square distance to preserve the original frequency data and to compensate for the different number of data points per language area in the current study and Kusakabe (1964). The languageby-language distances were used as input for multidimensional scaling (MDS). Figure 9 shows the MDS solution in two dimensions - Stress- $\mathrm{I}=.085$, indicating a fair to good fit (Kruskal 1964). The closer two points are in the figure, the more similar they are to each other.

Four things can be learned from Figure 9. First, the old colour systems of Miyako and Yaeyama were more similar to each other than they were to Standard Japanese. Second, the new colour systems of Miyako and Yaeyama are more similar to Standard Japanese than they are to their respective historic pasts. Third, while the new colour systems of Miyako and Yaeyama resemble each other more than they used to, they are still distinct. Finally, whereas the older colour systems of Miyako and Yaeyama were equally distinct from Standard Japanese, Miyako now resembles Japanese more than Yaeyama does. These last two points indicate that the two language areas have had their own trajectories towards their current forms. Nevertheless, the first two points seem to indicate that the changes have happened fairly rapidly.

7. In Kusakabe (1964), 24 speakers (27\%) used terms designated as 'other'.

8. While Kusakabe (1964) does not contain actual Japanese naming data, the Japanese data presented in Berlin \& Kay (1969) and subsequent studies (Uchikawa \& Boynton 1987; Kuriki et al., 2017) suggest negligible differences in the core set of responses for these colour chips. As such, we consider our newly collected Japanese data to closely resemble the Japanese colour system of the 1960 . 


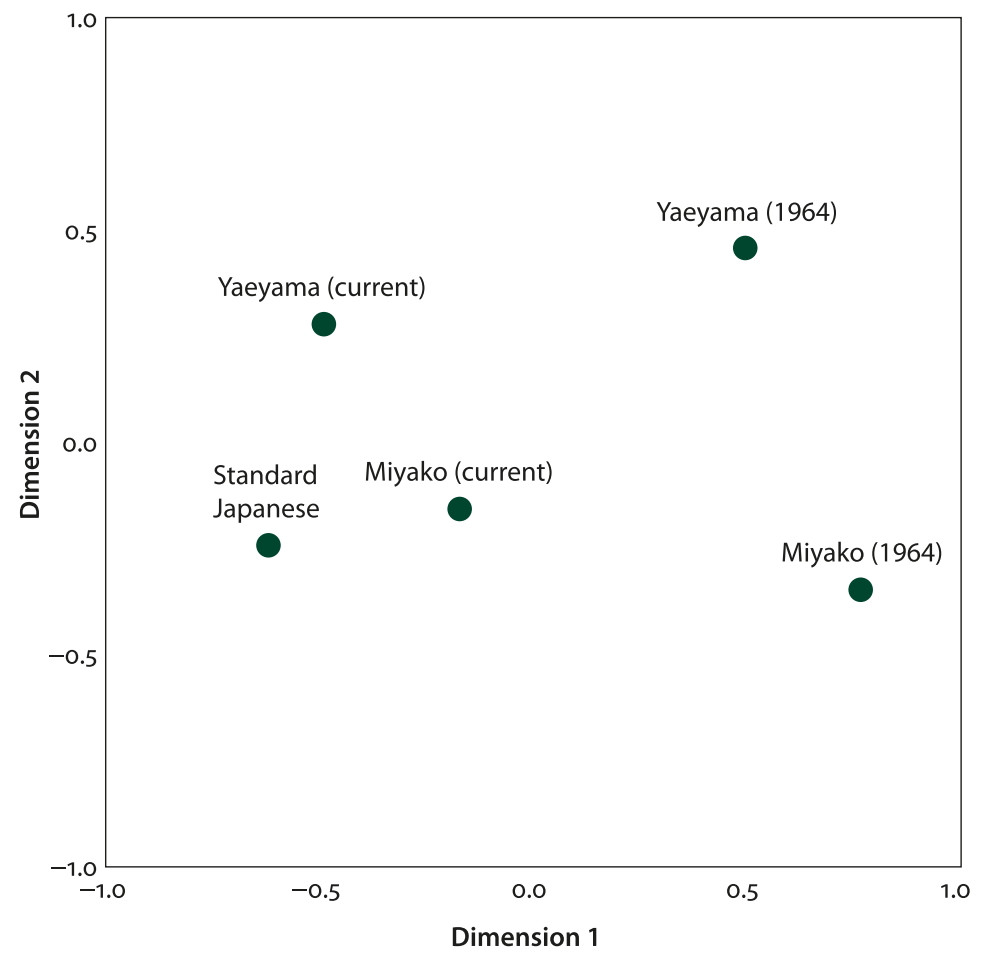

Figure 9. Multidimensional scaling solution for language similarity based on colour naming of 13 colour chips common between Kusakabe (1964) and the current study

\section{Discussion}

We set out to investigate the colour lexicon of contemporary Ryukyuan speakers, in the context of the many changes in the cultural and linguistic landscape in recent decades. While the colour lexicons of Ryukyuan and Japanese were examined in the 1960s (Berlin \& Kay 1969; Kusakabe 1964), subsequent research on colour terminology in the Japonic language family mainly concerned Standard Japanese (e.g., Kuriki et al. 2017; Uchikawa \& Boynton 1987). Statistical analysis of a core set of colour terms chosen for their longevity and widespread use across the language family showed that the Ryukyuan languages are distinct from Standard Japanese, hinting at the apparent stability of the colour system. This was most noticeable in the term ao for 'grue', which was used for a wider range encompassing green and blue across the Ryukyuan languages, whereas the term was only used for blue in contemporary Standard Japanese. Further evidence comes from the cognates of shiro and kuro which are used for achromatic 'white' and 'black' in Standard Japanese, but extend to 'light' and 'dark' in Ryukyuan, as has been 
hypothesised to be the case in pre-Old Japanese (Stanlaw 2007, 2010), again a sign of the longevity of the colour system.

At the same time, our naming data also exhibit considerable differences with previous data. A direct comparison between Kusakabe (1964) and the current study indicates that the modern Ryukyuan languages are closer to Standard Japanese than they are to their historic pasts. The responses elicited in the naming task of the current study revealed that several new terms, often standard(-like) phonological forms or loanwords, have entered Ryukyuan speakers' vocabulary through influence from both Standard Japanese (e.g., midori) and English (e.g., guriin). Most of these newly introduced terms were used for categories not previously reported for Ryukyuan, such as 'green' and 'orange' (Kusakabe 1964; but see also Hirayama 1992). Moreover, 'light blue' - not part of the original Berlin and Kay framework - has also emerged in two of the three Ryukyuan languages included in this study, mirroring recent developments in Standard Japanese (Uchikawa \& Boynton 1987; Kuriki et al. 2017).

However, the modal responses from the naming task revealed fewer salient colour categories in the Ryukyuan varieties overall, both when compared to our Standard Japanese data and previously reported Japanese data (e.g., Kuriki et al. 2017). Additional work is needed to determine which of the colour terms used in the Ryukyuan languages enjoy basic status, especially as the boundaries of newer categories - i.e., those not reported in Kusakabe (1964), such as 'green' and 'orange' - were less pronounced, with lower consensus between speakers shown by the absence of clear modal responses for chips in these parts of the colour spectrum. These findings likely represent variation between individual speakers resulting from ongoing changes. Individual members of a society can be at different stages of the evolutionary sequence of colour lexicons (MacLaury 1991). Ryukyuan speakers can have different levels of exposure to Japanese and English colour terms, which in turn influences their use, resulting in the variation we found in our data. However, further study is required to fully understand how different levels of exposure to other languages influences the use of colour terms (see, e.g., Athanasopoulos et al. 2011).

While it can be difficult to pinpoint the exact sources of specific new colour terms, there are several factors that could explain how they arise. First of all, if we assume that all speakers end up refining their division of the colour spectrum as technology and other cultural factors change over time, then all languages - including Ryukyuan - will eventually do so by themselves. Given the extensive dyeing practices in the Ryukyu Islands, one could have expected the languages spoken there to have more extensive colour lexicons earlier, so perhaps languages are only now catching up to the needs of the material culture. The fact that Ryukyuan speakers used several source-based terms specific to their 
local environment - and that sometimes referred to materials used in dyeing, such as fumbutu 'tree' - indicates that the languages were expanding their colour lexicon independently, at least to some degree. An interesting side note is the fact that Ryukyuan language areas did not group together in the same way for each of the colour cognate sets, showing that language-specific developments may affect older colour terms in different ways, which result in separate trajectories of semantic change for colour terms across a language family.

Secondly, the fact that there are no monolingual speakers of Ryukyuan today also needs to be considered. Bilingualism in itself has been shown to affect the stability of both the boundaries and foci of colour categories, with bilinguals showing more variation (Caskey-Sirmons \& Hickerson 1977; Jameson \& Alvarado 2007). In our case, speakers are bilingual in a standardised language with institutionalised education and an endangered minority language that was for the longest time seen as a dialect of Japanese (Heinrich 2005), and it is currently not well understood how bilingualism, language loss, and semantic change interact in such situations. Moreover, there might be effects that arise as a result of contact through specific language learning. Colour words are commonly part of primary school instruction and the words aka 'red', ao 'blue', shiro 'white', kuro 'black', kiiro 'yellow', midori 'green' and chairo 'brown', as well as their kanji characters, are learned by the third year of primary school. Such early exposure might be one of the reasons why many speakers use the Japanese form midori in the naming task.

Third, there could be a role for contact with foreign material culture - rather than language itself - that changes colour naming systems, as the cultural environment has been shown to alter colour naming patterns. A comparison of Japanese speakers in Germany, Tokyo and Yamagata Prefecture found that those living in Germany showed the strongest influence of Western culture. More importantly, speakers living in Tokyo - where there is relatively more exposure to Western culture - showed more influence than those living in Yamagata - where there is less exposure to Western culture (e.g., Iijima et al. 1982; Zollinger 1988). These results mainly pertained to the categorisation of colour, but contact with Western culture has also led to introduction of new terms. While this was not the case in our naming task data, several languages in the World Color Survey use source-based colour terms that are based on items introduced after Western influence, such as coffee and chocolate.

Another final dimension to consider in the case of endangered languages, such as Ryukyuan, is the social value of retaining tradition. As described above, one Miyako speaker insisted on the use of only four colour terms, even though they knew about the additional terms that other speakers were using. Several other speakers commented on the traditionally smaller colour lexicon of Ryukyuan compared to Japanese. Similar attitudes towards the conservation of 
language and culture have been shown to lead to the maintenance of "archaic" colour categories in, e.g., Navenchauc Tzotzil (see MacLaury 1991). Such attitudes can favour an older colour system and increase stability, which is an important factor to consider in the broader context of the notion of a constantly evolving and expanding colour lexicon (cf. Berlin \& Kay 1969).

\title{
5. Conclusion
}

To conclude, the interaction between standard and minority languages, and the modern backdrop of globalisation, has led to the use of new forms for new meanings by contemporary speakers of Ryukyuan (e.g., midori or guriin for 'green'). However, these new colour categories are not yet fully entrenched, indicating ongoing changes, and suggest the establishment of form precedes the establishment of meaning. A direct comparison with data from several decades ago shows that the modern Ryukyuan colour systems are closer to Standard Japanese than they are to their respective historic pasts, pointing to the speed at which semantic changes can occur. At the same time, analysis of four colour cognate sets reveals that recent Ryukyuan colour systems resemble each other more than they resemble Standard Japanese, hinting at the apparent stability of language-specific developments in semantics. Together, these findings highlight the need for additional, contemporary colour naming data to compare to historical data in related languages. Only then can we better understand the evolution of colour terms and their meaning more broadly.

\section{Funding}

This research was funded by the Nederlandse Organisatie voor Wetenschappelijk Onderzoek (NWO).

\section{Acknowledgements}

We would like to thank all speakers who participated in the study.

\author{
Abbreviations \\ CONT continuous \\ DAT dative \\ GEN genitive \\ NPST non-past
}




\section{References}

Athanasopoulos, Panos. 2009. Cognitive representation of colour in bilinguals: The case of Greek blues. Bilingualism: Language and Cognition 12(1). 83-95. https://doi.org/10.1017/S136672890800388X

Athanasopoulos, Panos, Ljubica Damjanovic, Andrea Krajciova \& Miho Sasaki. 2011. Representation of colour concepts in bilingual cognition: The case of Japanese blues. Bilingualism: Language and Cognition 14(1). 9-17. https://doi.org/10.1017/S1366728909990046

Berlin, Brent \& Paul Kay. 1969. Basic color terms: Their university and evolution. California University Press. Berkeley.

Caskey-Sirmons, Leigh A. \& Nancy P. Hickerson. 1977. Semantic shift and bilingualism: Variation in the color terms of five languages. Anthropological Linguistics 19(8). 358-367.

Cattell, Raymond B. 1966. The scree test for the number of factors. Multivariate Behavioral Research 1(2). 245-276. https://doi.org/10.1207/s15327906mbro102_10

Conlan, Francis. 2005. Searching for the semantic boundaries of the Japanese colour term 'ao'. Joondalup: Edith Cowan University $\mathrm{PhD}$ dissertation.

Ember, Melvin. 1978. Size of color lexicon: Interaction of cultural and biological factors. American Anthropologist 8o(2). 364-367. https://doi.org/10.1525/aa.1978.80.2.02a00100

Haynie, Hannah J. \& Claire Bowern. 2016. Phylogenetic approach to the evolution of color term systems. Proceedings of the National Academy of Sciences 113(48). 13666-13671. https://doi.org/10.1073/pnas.1613666113

Heinrich, Patrick. 2005. What leaves a mark should no longer stain. In Mike Evans (ed.), Refereed Papers from the 1st International Small Islands Cultures Conference, 61-72. Sydney: Small Island Culture Research Initiative.

Heinrich, Patrick, Shinsho Miyara \& Michinori Shimoji. 2015. Introduction: Ryukyuan languages and Ryukyuan linguistics. In Patrick Heinrich, Shinsho Miyara \& Michinori Shimoji (eds.), Handbook of the Ryukyuan languages. History, structure, and use, 1-10. Berlin: De Gruyter Mouton. https://doi.org/10.1515/9781614511151.1

Hendrick-Wong, Esme. 2013. Purple mania: How a snail created the most sought after colour of all time. Textiles Asia 4(3). 7-13.

Hirayama, Teruo. 1992. Gendai Nihongo Hōgen Daijiten [Dictionary of contemporary Japanese dialects]. Tokyo: Meiji-shoin.

Huisman, John L.A., Asifa Majid \& Roeland van Hout. 2019. The geographical configuration of a language area influences linguistic diversity. PLoS ONE 14(6). e0217363. https://doi.org/10.1371/journal.pone.0217363

Iijima, Toshiro, Wolfgang Wenning \& Heinrich Zollinger. 1982. Cultural factors of color naming in Japanese: Naming tests with Japanese children in Japan and Europe. Anthropological Linguistics 24(2). 245-262.

Iwasaki, Shoichi. 2013. Japanese. Amsterdam: John Benjamins. https://doi.org/10.1075/loall.17

Jäger, Gerhard. 2012. Using statistics for cross-linguistic semantics: A quantitative investigation of the typology of colour naming systems. Journal of Semantics 29(4). 521-544. https://doi.org/10.1093/jos/ffsoo6

Jameson, Kimberley A. \& Nancy Alvarado. 2003. Differences in color naming and color salience in Vietnamese and English. Color Research \& Application 28. 113-138. https://doi.org/10.1002/col.10131 
Kaiser, Henry F. 196o. The application of electronic computers to factor analysis. Educational and Psychological Measurement 20(1). 141-151. https://doi.org/10.1177/001316446002000116

Kay, Paul, Brent Berlin, Luisa Maffi \& William R. Merrifield. 1997. Color naming across languages. In Clyde L. Hardin \& Luisa Maffi (eds.), Color categories in thought and language, 21-56. Cambridge: Cambridge University Press. https://doi.org/10.1017/CBO9780511519819.002

Kay, Paul, Brent Berlin, Luisa Maff, William R. Merrifield \& Richard S. Cook. 2009. The world color survey. Stanford: CSLI Publications.

Kay, Paul, Brent Berlin \& William Merrifield. 1991. Biocultural implications of systems of color naming. Journal of Linguistic Anthropology 1(1). 12-25. https://doi.org/10.1525/jlin.1991.1.1.12

Kristol, Andres M. 1980. Color systems in southern Italy: A case of regression. Language 56(1). 137-145. https://doi.org/10.2307/412646

Kruskal, Joseph B. 1964. Multidimensional scaling by optimizing goodness of fit to a nonmetric hypothesis. Psychometrika 29(1). 1-27. https://doi.org/10.1007/BF02289565

Kuriki, Ichiro, Ryan Lange, Yumiko Muto, Angela M. Brown, Kazuho Fukuda, Rumi Tokunaga, Delwin T. Lindsey, Keiji Uchikawa \& Satoshi Shioiri. 2017. The modern Japanese color lexicon. Journal of Vision 17(3). 1-18. https://doi.org/10.1167/17.3.1

Kusakabe, Fumio. 1964. Aka to Ao - Okinawa, Miyako, Yaeyama o megutte [Red and blue around Okinawa, Miyako and Yaeyama]. Kokugogaku 58. 60-75.

Levinson, Stephen C. 200o. Yeli Dnye and the theory of basic color terms. Journal of Linguistic Anthropology 10(1).3-55. https://doi.org/10.1525/jlin.2000.10.1.3

Lillo, Julio, Fernando González-Perilli, Lilia Prado-León, Anna Melnikova, Leticia Álvaro, José A. Collado \& Humberto Moreira. 2018. Basic color terms (BCTs) and categories (BCCs) in three dialects of the Spanish language: Interaction between cultural and universal factors. Frontiers in Psychology 9. 761. https://doi.org/10.3389/fpsyg.2018.00761

Lucy, John A. 1997. The linguistics of color. In Clyde L. Hardin \& Luisa Maffi (eds.), Color categories in thought and language, 320-346. Cambridge: Cambridge University Press. https://doi.org/10.1017/CBO9780511519819.015

MacLaury, Robert E. 1986. Color in Meso-America: Vol I. A theory of composite categorization. Berkeley: University of California $\mathrm{PhD}$ dissertation.

MacLaury, Robert E. 1991. Social and cognitive motivations of change: Measuring variability in color semantics. Language 67(1). 34-62. https://doi.org/10.2307/415538

MacLaury, Robert E. 2001. Color terms. In Martin Haspelmath, Ekkehard König, Wulf Oesterreicher \& Wolfgang Raible (eds.), Language typology and language universals, 1227-1251. Berlin: Walter de Gruyter.

MacLaury, Robert E., Gordon W. Hewes, Paul R. Kinnear, Jan B. Deregowski, William R. Merrifield, Barbara A.C. Saunders, James Stanlaw, Christina Toren, J. Van Brakel \& Roger W. Wescott. 1992. From brightness to hue: An explanatory model of color-category evolution [and comments and reply]. Current Anthropology 33(2). 137-186. https://doi.org/10.1086/204049

Majid, Asifa, Fiona Jordan \& Michael Dunn. 2011. Evolution of semantic systems: Procedures manual. Nijmegen: Max Planck Institute for Psycholinguistics.

Majid, Asifa, Fiona Jordan \& Michael Dunn. 2015. Semantic systems in closely related languages. Language Sciences 49(1). 1-18. https://doi.org/10.1016/j.langsci.2014.11.002

Miyara, Toso. 1930. Yaeyama Goi [Yaeyama vocabulary]. Tokyo: Toyo Bunko.

Naroll, Raoul. 1970. What have we learned from cross-cultural surveys? American Anthropologist 72(6). 1227-1288. https://doi.org/10.1525/aa.1970.72.6.02a0o030 
Özgen, Emre \& Ian R.L. Davies. 1998. Turkish color terms: tests of Berlin and Kay's theory of color universals and linguistic relativity. Linguistics 36(5). 919-956. https://doi.org/10.1515/ling.1998.36.5.919

Paramei, Galina V. 2005. Singing the Russian blues: An argument for culturally basic color terms. Cross-Cultural Research 39(1). 10-38. https://doi.org/10.1177/1069397104267888

Pellard, Thomas. 2015. The linguistic archeology of the Ryukyu Islands. In Patrick Heinrich, Shinsho Miyara \& Michinori Shimoji (eds.), Handbook of the Ryukyuan languages. History, structure, and use, 13-38. Berlin: De Gruyter Mouton. https://doi.org/10.1515/9781614511151.13

Regier, Terry, Paul Kay \& Naveen Khetarpal. 2007. Color naming reflects optimal partitions of color space. Proceedings of the National Academy of Sciences 104(4). 1436-1441. https://doi.org/10.1073/pnas.0610341104

Regier, Terry, Paul Kay \& Naveen Khetarpal. 2009. Color naming and the shape of color space. Language 85(4): 884-892. https://doi.org/10.1353/lan.0.0177

Shibatani, Masayoshi. 1990. The languages of Japan. Cambridge: Cambridge University Press.

Shogakukan (200o). Nihon Kokugo Daijiten [Unabridged Dictionary of the Japanese Language]. Tokyo: Shogakukan.

Stanlaw, James. 1987. Colors, culture, and contact: English loanwords and problems of color nomenclature in modern Japanese. Champaign: University of Illinois PhD dissertation.

Stanlaw, James. 2007. Japanese color terms, from 400 CE to the present: Literature, orthography, and language contact in light of current cognitive theory. In Robert E. MacLaury, Galina V. Paramei \& Don Dedrick (eds.), Anthropology of color: Interdisciplinary multilevel modeling, 295-318. Amsterdam: John Benjamins. https://doi.org/10.1075/z.137.18sta

Stanlaw, James. 2010. Language, contact, and vantages: fifteen hundred years of Japanese color terms. Language Sciences 32(2). 196-224. https://doi.org/10.1016/j.langsci.2009.10.005

Tsuhako, Toshiko. 1992. Okinawa Chūnanbu Hōgen [Dialects of central and southern Okinawa]. In Takashi Kamei, Rokuro Kono \& Eiichi Chino (eds.), Nihon Rettō no Gengo [Languages of the Japanese archipelago], 829-848. Tokyo: Sanseido.

Uchikawa, Keiji \& Robert M. Boynton. 1987. Categorical color perception of Japanese observers: Comparison with that of Americans. Vision Research 27(10). 1825-1833. https://doi.org/10.1016/0042-6989(87)90111-8

Wierzbicka, Anna. 2008. Why there are no 'colour universals' in language and thought. Journal of the Royal Anthropological Institute 14(2). 407-425. https://doi.org/10.1111/j.1467-9655.2008.00509.x

Zollinger, Heinrich. 1988. Categorical color perception: Influence of cultural factors on the differentiation of primary and derived basic color terms in color naming by Japanese children. Vision Research 28(12). 1379-1382. https://doi.org/10.1016/0042-6989(88)90069-7 